\title{
How Enzymes Work: A Look through the Perspective of Molecular Viscoelastic Properties
}

\author{
Hao Qu and Giovanni Zocchi* \\ Department of Physics and Astronomy, University of California Los Angeles, Los Angeles, California 90095-1547, USA
}

(Received 24 January 2012; published 1 February 2013)

\begin{abstract}
We present nanorheology measurements on the folded state of an enzyme that show directly that the (ensemble-averaged) stress-strain relations are nonlinear and frequency dependent beyond 1 - $\AA$ deformation. We argue that this frequency dependence allows for opening a nonequilibrium cycle in the forcedeformation plane if the forward and backward conformational changes of the enzyme during catalysis happen at different speeds. Using a heuristic model for the experimentally established viscoelastic properties of the enzyme, we examine a number of general features of enzymatic action. We find that the proposed viscoelastic cycle is consistent with the linear decrease of the speed of motor proteins with load. We find a relation between the stall force and the maximum rate for enzymes (in general) and motors (in particular). We estimate the stall force of the motor protein kinesin from thermodynamic quantities and estimate the maximum rate of enzymes from purely mechanical quantities. We propose that the viscoelastic cycle provides a framework for considering mechanochemical coupling in enzymes on the basis of possibly universal materials properties of the folded state of proteins.
\end{abstract}

DOI: 10.1103/PhysRevX.3.011009

Subject Areas: Biological Physics, Chemical Physics, Statistical Physics

\section{INTRODUCTION}

Enzymes are different from passive catalysts: They are thermodynamic machines at molecular scale. This is obvious from the familiar examples of motor proteins and ion pumps, but, in general, mechanochemical coupling is central to the working of all enzymes [1-9]. The mechanisms of action of many enzymes are known in detail in structural terms [10]; the canonical description of the dynamics is through the Michaelis-Menten approach, which, in its most basic form, describing enzymatic processes in terms of rate equations:

$$
S+E \stackrel{k_{-1}, k_{1}}{\longrightarrow} C \stackrel{k_{-2}, k_{2}}{\longrightarrow} P+E,
$$

where $S$ stands for substrate(s), $E$ for enzyme, $P$ for product(s), and $C$ for the enzyme-substrate complex. This description can be complicated by adding more intermediate states, but in this paper we argue that this approach misses one fundamental aspect, namely, that, from left to right in Eq. (1), the enzyme goes through a thermodynamic cycle. That is, the enzyme moves from an initial equilibrium state $A$ to some (in general, nonequilibrium) state $B$ and back to $A$, but the return path $B \rightarrow A$ in phase space is different from the time reverse of $A \rightarrow B$. This statement is obvious in the case of motor proteins, because no work can be extracted from a system that goes back and forth along the same trajectory in phase space (for example, an oscillating spring). Here, we propose that this notion holds, in

\footnotetext{
*zocchi@physics.ucla.edu
}

Published by the American Physical Society under the terms of the Creative Commons Attribution 3.0 License. Further distribution of this work must maintain attribution to the author(s) and the published article's title, journal citation, and DOI. general, for enzymes, and we give an explicit representation of the cycle in question which, we suggest, has universal features common to all enzymes. From this we draw several conclusions about the dynamics of enzymes, which are seen to be consistent with experimental observations.

The basis for our proposal is the viscoelastic transition of the folded state of proteins, which we discovered recently [11]. The experiments are performed in the setup shown in Fig. 1, where gold nanoparticles (GNPs) are tethered to a gold surface by the enzyme under study through cysteine residues. We estimate that the average number of guanylate kinase (GK) tethers per GNP is 3 , and the measurements are averaged over approximately $10^{7}$ GNPs [11]. An AC electric field orthogonal to the surface drives the negatively charged GNPs, while the amplitude of the response of the GNPs, averaged over many GNPs, is measured by evanescent wave scattering in a phase-locked loop [12]. The response amplitude, $z$, is the amplitude of the collective oscillation of the GNPs at the driving frequency. Response amplitudes as small as a fraction of $1 \AA$ can be measured for frequencies between $10 \mathrm{~Hz}$ and $10 \mathrm{kHz}$, giving access to the rheological properties of the folded state of the enzyme in this frequency range. By rheological properties, we mean the mechanical properties characterizing the ensembleaveraged dynamics directly measured in the experiments. For the enzyme GK, we find a reversible transition from elastic to viscoelastic behavior as the amplitude of the forcing $|F|$ is increased [11]. At a fixed frequency, the force-deformation curve is piecewise linear, indicating a conformational "softening" in the enzyme beyond a yield deformation $\left(z_{\mathrm{c}} \approx 1 \AA\right.$ at $10 \mathrm{~Hz}$ [13], where the subscript "c" stands for "critical"). This softening is shown in Fig. 2(a), which displays measurements of the amplitude of the deformation $|z|$ obtained at $\nu=10 \mathrm{~Hz}$ forcing frequency for varying amplitudes of the forcing (proportional 


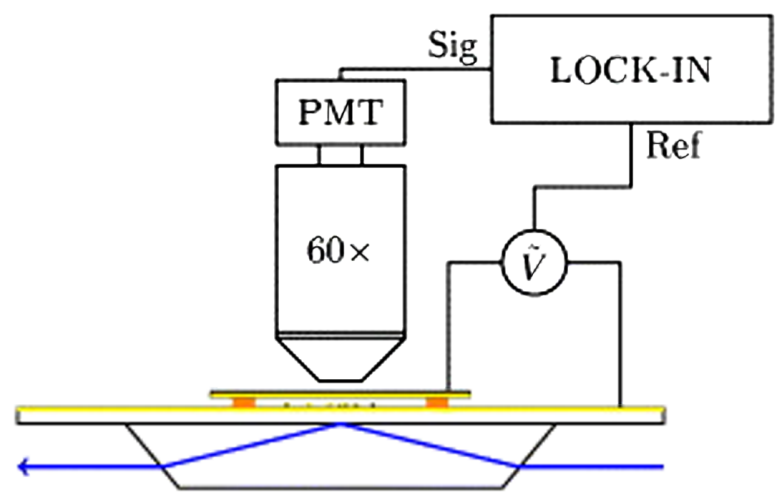

(a)

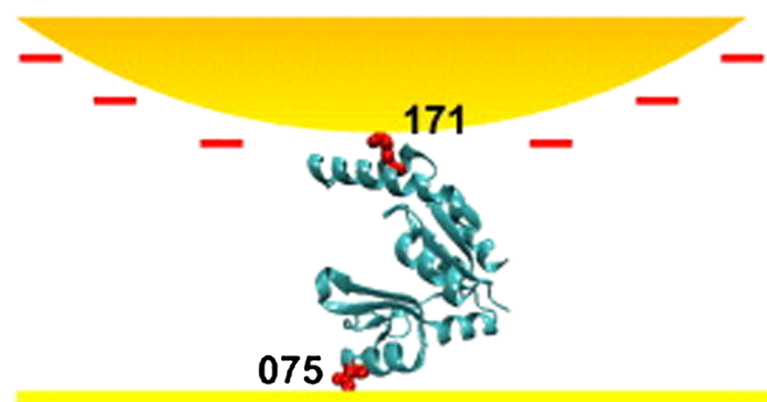

(b)

FIG. 1. The experimental system with which the data of Fig. 2 were obtained. (a) The schematics of the sample chamber with AC electrophoretic excitation and synchronous evanescentwave-scattering optical measurement of displacement. "Sig" stands for signal, "PMT" for photomultiplier, and "Ref" for reference. (b) Geometry of the enzyme GK tethering a GNP to the gold-coated slide. The attachment points (residues mutated to cysteins at positions 171 and 75) are shown on the structure. The enzyme and the 20-nm-diameter GNP are drawn approximately to scale.

to the applied voltage $V$ ). On the other hand, at fixed forcing amplitude, the behavior in frequency is characteristic of linear viscoelasticity, as shown in Fig. 2(c): The deformation amplitude is constant at high frequency (the behavior of a spring) and diverges as $1 / \omega$ at low frequencies (the behavior of a viscous flow) [10]. The break in the curve of Fig. 2(a) defines a yield deformation $z_{c}$ (and a corresponding yield force $F_{\mathrm{c}}$ ); however, this quantity must be frequency dependent, and, in fact, increases with $\nu$. This behavior follows from general considerations on the dependence of the strength of molecular bonds on the pulling rate [14], and is confirmed by the experiments. Indeed, Fig. 2(b) shows two force-deformation curves measured for the same sample at two different forcing frequencies (10 and $100 \mathrm{~Hz}$ ). Both curves are roughly piecewise linear, as in Fig. 2(a), but the break in the slope (the transition from the linear to the nonlinear regime) occurs at a higher critical force (and critical deformation $z_{c}$ ) for the $100-\mathrm{Hz}$ curve.
The two curves coincide in the (first) linear regime of the $10-\mathrm{Hz}$ curve (showing that this is indeed a frequencyindependent "simple-spring" regime), but this simplespring regime is extended to larger $z$ for the $100-\mathrm{Hz}$ curve. That is, the (first) linear regime, where the deformation is proportional to the force, survives up to larger deformations at higher frequencies. This experimental fact, namely that the linear elasticity regime extends to larger deformations at higher frequencies, is a new experimental finding not previously reported. We propose that the physical basis of this phenomenon is similar to the Evans-Ritchie result [14] for the pulling-rate dependence of the strength of molecular bonds. In order to get out of the linear-elasticity regime, the system has to climb over a barrier, and because of thermal fluctuations, there is a frequency dependence in the critical force (measured in ensemble-averaged experiments) at which this transition happens. Beyond the corresponding viscoelastic transition (to the right of the break in the slope in each of the force-deformation curves shown in Fig. 2(b)), the slopes of the $10-$ and $100-\mathrm{Hz}$ curves are again similar if not identical, a result which constrains the possible viscoelastic models that can account for these data. Finally, the curves in Fig. 2 represent transformations between nonequilibrium states that are reversible, as we already mentioned in [11]. The measurements in Fig. 2(b) were obtained in the temporal sequence: $10-\mathrm{Hz}$ run $1 \rightarrow 100-\mathrm{Hz}$ run $\rightarrow 10-\mathrm{Hz}$ run 2 , for the same sample, so the filled circles show that after the $100-\mathrm{Hz}$ run, which displays a higher $z_{c}$, one can come back to essentially the initial $10-\mathrm{Hz}$ response curve.

Let us summarize our understanding of the viscoelastic transition [10], based on the measurements published in $[10,11]$ and the new measurements shown in Fig. 2. At a fixed forcing amplitude, the response (the deformation $z$ ) is frequency dependent, constant at high frequencies, and diverging as $1 / \omega$ at low frequencies. The $z$ vs $\omega$ curve is well represented by the Maxwell model of linear viscoelasticity. At a fixed frequency, the force-deformation curves are piecewise linear, defining a frequencydependent yield deformation $\left(z_{c}\right)$ and force (Fig. 2). The location of the yield transition moves slowly with frequency, as is evident from Fig. 2(b). In the following discussion, we assume that the same qualitative features of the force-deformation curves (i.e., the existence of the yield transition) shown in Fig. 2(b) are present at the higher frequencies that correspond to the time scale of this enzyme's action (approximately $1 \mathrm{kHz}$ ). In the experimental setup, we are limited in exploring the yield transition at higher frequencies by the limited force that we can apply to the GNPs and also by the frequency cutoff due to the hydrodynamic dissipation of the GNPs.

\section{HYPOTHESIS}

The viscoelastic transition is the starting point of our analysis. We consider the "stress-strain" diagram (actually 
(a)

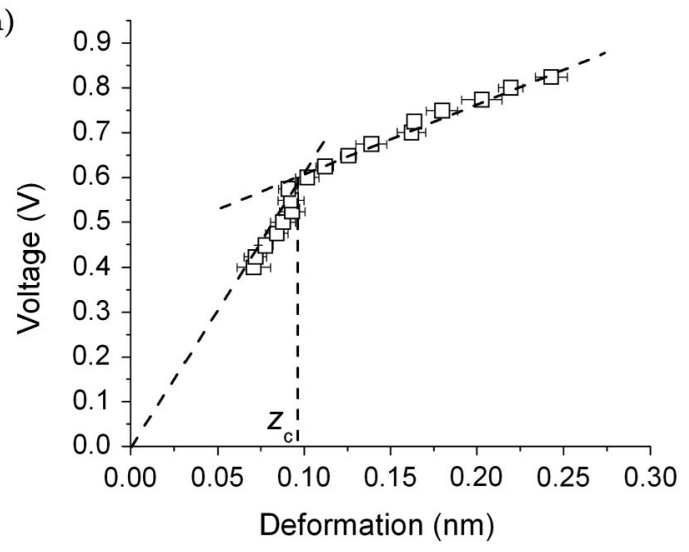

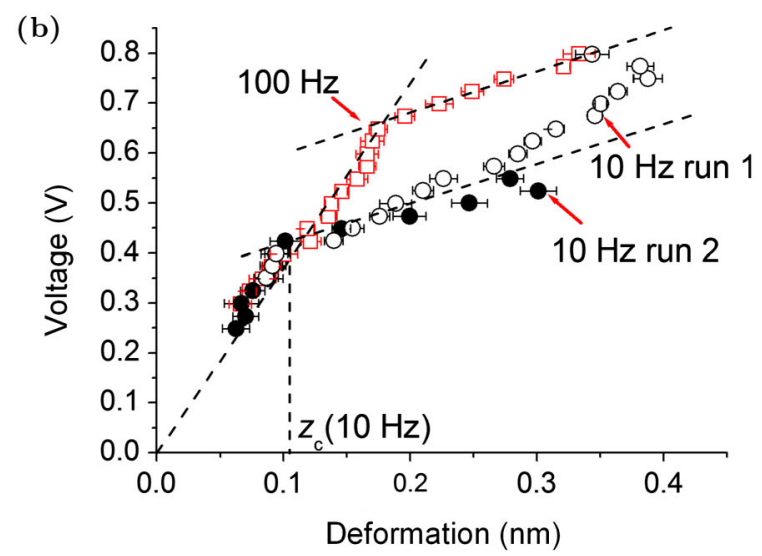

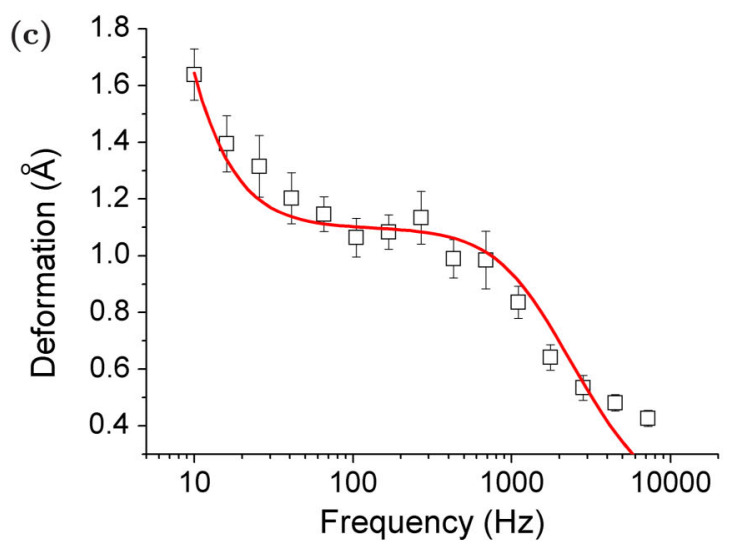

FIG. 2. The (ensemble-averaged) AC-driven mechanical response of GK. The amplitude of the oscillating force applied to the GNPs is proportional to the voltage; the horizontal axis is the measured amplitude of the resulting deformation. (a) The force-deformation curve is piecewise linear, the break in the slope occurring at a "yield" deformation $z_{\mathrm{c}} \sim 1 \AA$, where $z$ is the deformation of GK. To the left of this transition is the linear-elasticity regime (the straight line extrapolating to the origin), where the protein behaves like a simple spring. To the right of the transition lies a viscoelastic regime where the protein essentially flows like a viscous fluid [11,13]. The transition is reversible. (b) The mechanical response as in (a) measured at two different frequencies (10 Hz, empty and filled circles, and $100 \mathrm{~Hz}$, red squares) for the same sample [which is a different sample from the one in (a)]. These measurements show that the viscoelastic transition (the yield strain $z_{c}$ ) is frequency dependent. For the $100 \mathrm{~Hz}$ curve, the linear-elasticity regime is extended up to larger deformations. The $100-\mathrm{Hz}$ and $10-\mathrm{Hz}$ curves coincide in the linear-elasticity region of the $10-\mathrm{Hz}$ curve, confirming that this is indeed a frequency-independent "simple-spring" regime. The three curves were obtained in the sequence $10-\mathrm{Hz}$ run $1,100 \mathrm{~Hz}, 10-\mathrm{Hz}$ run 2. Thus, the filled circles show that these measurements explore nonequilibrium but reversible processes. (c) Amplitude of the response vs frequency for GK at fixed force. The line shows a fit to the simplest description of linear viscoelasticity (Maxwell model) [10]. The drop above $1 \mathrm{kHz}$ is due to the hydrodynamic friction on the GNPs. The protein shows the behavior of a spring between 100 $\mathrm{Hz}$ and $1 \mathrm{kHz}$ (the plateau) and that of a viscous fluid below $100 \mathrm{~Hz}$ (the divergence).

a force-deformation diagram) of Fig. 2. For different frequencies $\nu$, one obtains different curves: This fact opens the possibility of a cycle enclosing a nonzero area in the force-deformation plane if the forward and backward conformational changes of the enzyme happen at different speeds. Here and throughout the paper, we are describing the ensemble-averaged dynamics of the system. The measurements of Fig. 2 represent the ensemble-averaged mechanical response of the protein. Similarly, when we talk of the "speed" of the conformational change, we mean the ensemble-averaged speed of the process; individual trajectories fluctuate, of course. Figure 3 shows schematically our proposal for a nonequilibrium cycle describing enzyme action: $F$ represents the internal stress of the enzyme, $z$ the appropriately identified, pertinent conformational variable; $F=z=0$ defines the reference equilibrium state. We imagine that the process $A \rightarrow B \rightarrow C \rightarrow D$ is driven by substrate binding; $D \rightarrow E$ corresponds to the chemical reaction and/or release of the products; the return path of the cycle $E \rightarrow B \rightarrow A$ is driven by the enzyme's own restoring force (i.e., its internal stress, proportional to the gradient of the free energy). The leg $B \rightarrow C \rightarrow D$ is fast [corresponding to a frequency $\omega_{2}>\omega_{1}$ in Fig. 2(b)] and the leg $E \rightarrow B$ is slow (corresponding to a frequency $\omega_{1}$ ); i.e., in time, the cycle proceeds as represented schematically in Figs. 3(b) and 3(c). $A \rightarrow B, B \rightarrow C$ are in the elastic regime of the enzyme; $C \rightarrow D$ and $E \rightarrow B$ are in the viscoelastic regime. 
(a)

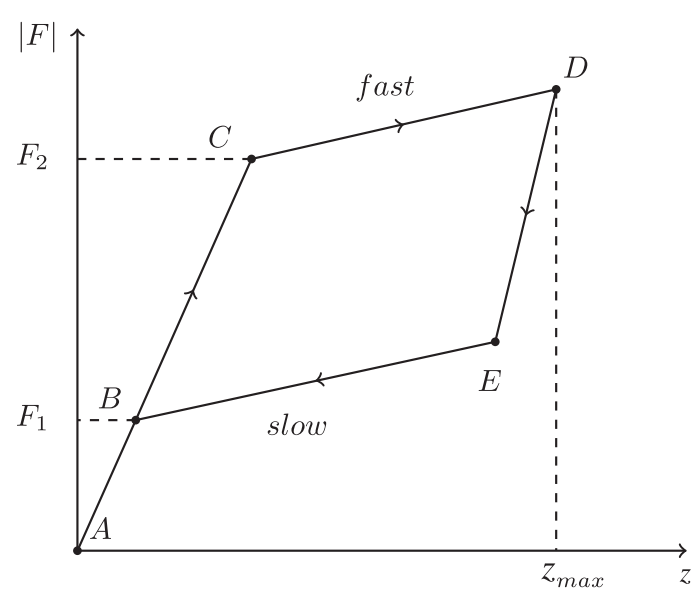

(b)

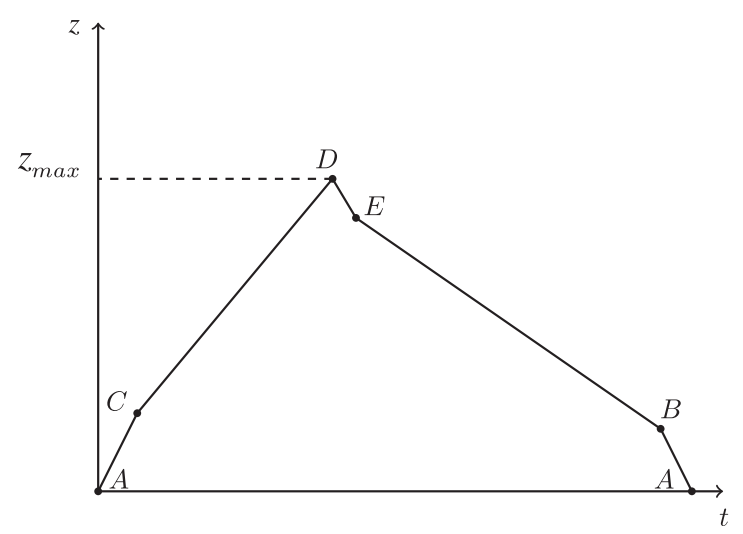

(c)

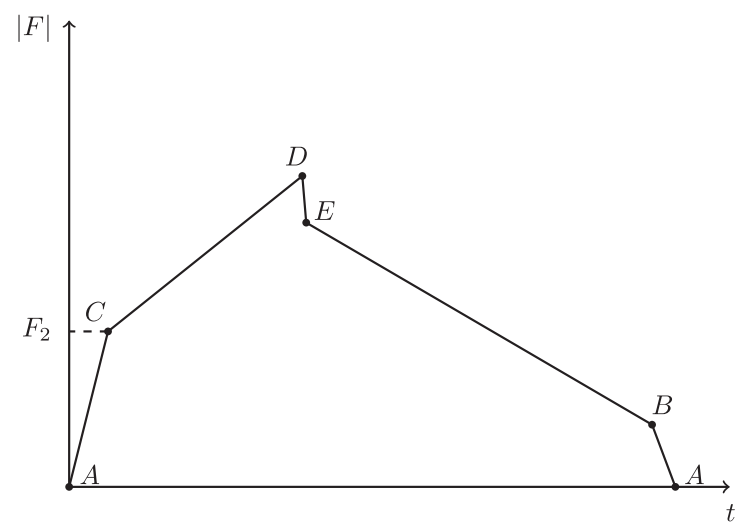

FIG. 3. Schematic diagrams illustrating the proposed nonequilibrium cycle of enzymes. $|F|, z$, and the trajectories $z(t)$ are ensemble-averaged quantities. (a) $|F|$ is the "internal stress" of the enzyme, $z$ the deformation. In this plane, a cycle opens if the forward conformational change is fast and the backward conformational change is slow. In this picture, the substrate binds the (open) form of the enzyme $A$; the process $A \rightarrow B \rightarrow C \rightarrow D$ is driven by the forces between the substrate and the enzyme and is fast. $D \rightarrow E$ corresponds to the chemical reaction and/or product release; the process $E \rightarrow B \rightarrow A$ is driven by the internal restoring force of the enzyme (proportional to the free-energy gradient) and is slow. In (b) and (c), we sketch the corresponding time dependence of the deformation ("reaction coordinate") $z$ and the internal stress $|F|$.
Let us go through the cycle depicted in Fig. 3(a) in somewhat more detail. For definiteness, we will think of the enzyme GK, for which the data of Fig. 2 were obtained and for which a detailed conformational and mechanical analysis was performed in [15]. Binding of the substrate guanosine monophosphate (GMP) drives the large "opento-closed" conformational change [7,15], which in the diagram of Fig. 3(a) corresponds to $A \rightarrow B \rightarrow C \rightarrow D$. This process is "externally" driven by the forces between substrate and enzyme, and is "fast"; it leaves the enzyme in the stressed state $D . D \rightarrow E$ corresponds to the reaction, which, in this case, is the transfer of the phosphate from adenosine triphosphate (ATP) to GMP. The very informative analysis in [15] shows that, in this step, the enzyme's conformational change is small, but internal stresses change, as do the forces between the enzyme and the ligands. For example, some parts of the enzyme are stiffer after the reaction step than they were before, other parts are floppier, and the distribution of stresses is, in general, different. We schematize this step, in which stresses change at relatively fixed conformation, with the segment $D \rightarrow E$ in the diagram in Fig. 3(a). This picture is supported, in the case of GK, by molecular dynamics simulation [15]. For example, the simulations show that some parts of the enzyme are stiffer. The internal stress partially "released" in $D \rightarrow E$ shows in the fact that the catalytic rates of the forward $\left(k_{\text {cat }}\right)$ and reverse $\left(k_{\text {cat }}^{\prime}\right)$ reactions are different. For GK, $k_{\mathrm{cat}} \approx 394 \mathrm{~s}^{-1}$, while $k_{\mathrm{cat}}^{\prime} \approx 90 \mathrm{~s}^{-1}$ [16].) The return path $E \rightarrow B \rightarrow A$ is driven by the internal restoring force of the enzyme (which can be significantly smaller than the driving force that originates from substrate binding) and is "slow": The products are released at $E$ or somewhere along $E \rightarrow B$, and the ground state of the enzyme without ligands is $A$. Below, we explore some consequences of this proposal of how enzymes work.

However, before we proceed, our respect for the opinions of the community compels us to discuss why and in what way we are departing from the traditional description. The traditional approach assumes a simplified "microscopic" energy landscape for the protein, usually consisting of two potential wells, and certain expressions (or, simply, values) for the rates of switching between wells, based on either the Kramers [17] or the Bell [18] theory. Dissipation is accounted for implicitly in the form of these rates. For example, the most popular description of molecular motors [19] treats a motor itself as a two-state system (while the interaction between the motor and the track is treated through the formalism of Fokker-Planck equations). However, the two-state picture is an idealization: A real system of enzymes accesses a large (but finite) number of different states [20,21]. We propose a description in terms of the opposite limit-an infinite number (a continuum) of states-and then we write down a heuristic equation of motion for the ensemble-averaged coordinate describing this motion, with the dissipation put in directly. 
This is a sensible approach which leads to predictions that can be directly related to our experimental data.

In fact, many ideas expressed in this paper have been put forward before, in work by Blumenfeld starting in 1971 [22,23], and summarized, for example, in [4]. In Blumenfeld's "relaxation model" of enzyme catalysis, the overall rate of the enzymatic cycle is determined by a conformational relaxation rate of the enzyme structure. (More recent experimental evidence supporting this view is contained, for example, in [24].) The enzyme structure exhibits long-lived nonequilibrium states during the catalytic cycle, and rates for the forward and backward reaction are, in general, different. These same ideas are reflected in the present model, which in addition contains an explicit representation of the mechanical "relaxation" cycle based on the experimental data now available on the viscoelastic transition of enzyme dynamics [25]. Indeed, this paper can be thought of as fulfilling, to some extent, Blumenfeld's program as expressed in [26], where we read the following statement in his conclusion: " ... the problem of developing a quantitative physical theory of enzymatic catalysis is reduced to that of developing a quantitative kinetic theory for conformational relaxation of protein macromolecules."

\section{DEDUCTIONS}

In discussing this system, it is useful to have some actual mechanical model in mind which, though heuristic, displays some of the relevant materials properties of the enzyme. The relevant property here is viscoelasticity, since, judged by the data of Fig. 2, most of the approximately $10-\AA$ conformational change accompanying catalysis in this enzyme happens in the viscoelastic regime. In this respect, some appropriate variant of the classic Maxwell model of viscoelasticity [consisting of a spring and a dashpot series as schematically illustrated in Fig. 4(a)] may play a role for the enzyme cycle analogous to the ideal gas for the Carnot engine. We therefore make our consideration based on this model: a spring of stiffness $k$, a dashpot with dissipation coefficient $\gamma$, and force $f$ applied to the cylinder of the dashpot. Once again, this model represents the ensemble-averaged mechanics of the enzyme at fixed (room) temperature, not the microscopic mechanics. Denoting the position of the piston and cylinder of the dashpot by $z_{1}, z_{2}$, respectively, the equation of motion for these two degrees of freedom is

$$
\left\{\begin{array} { l } 
{ \gamma \frac { d } { d t } ( z _ { 1 } - z _ { 2 } ) = k z _ { 1 } } \\
{ f = \gamma \frac { d } { d t } ( z _ { 1 } - z _ { 2 } ) }
\end{array} \Rightarrow \left\{\begin{array}{l}
\dot{z}_{2}=\frac{\dot{f}}{k}+\frac{f}{\gamma}, \\
z_{1}=\frac{f}{k},
\end{array}\right.\right.
$$

where $\dot{z}=d z / d t$ and $\dot{f}=d f / d t$.

We imagine the enzymatic cycle to proceed in the following way: (1) A constant external force $f_{0}$ acts for a time $t_{0}$. (This force originates from the substrate binding at $t=0$; at time $t_{0}$, the chemical reaction and/or the product release occurs.) (2) A constant restoring force $f_{1}$ acts until the enzyme is back in its equilibrium state at time $t_{0}+t_{1}$. (This action is the result of the free-energy gradient as the enzyme is displaced from the equilibrium state.) That is [see Fig. 4(b)],

$$
f(t)= \begin{cases}f_{0}-f_{1} & \text { for } 0 \leq t<t_{0} \\ -f_{1} & \text { for } t_{0} \leq t<t_{0}+t_{1} \\ 0 & \text { otherwise }\end{cases}
$$

Obviously, this equation is a schematization, grossly approximate in the details (for instance, physically, we must have $f_{1} \propto z_{2}$ for $z_{2}$ small enough) but reasonable in

(a)

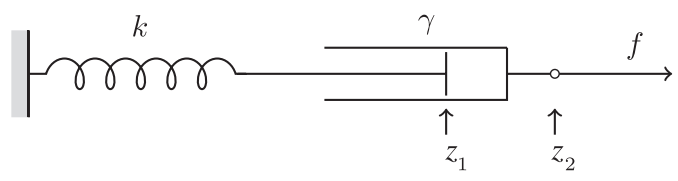

(b)

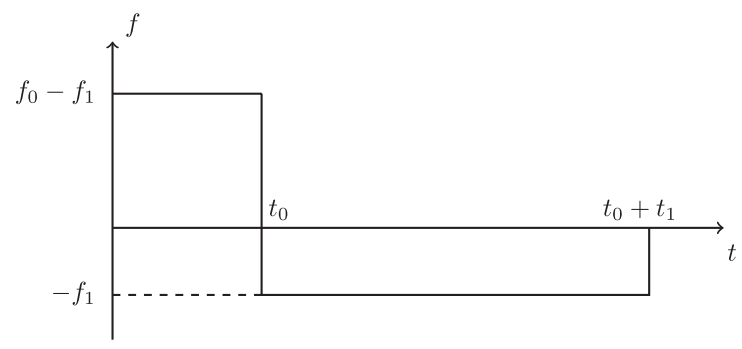

(c)

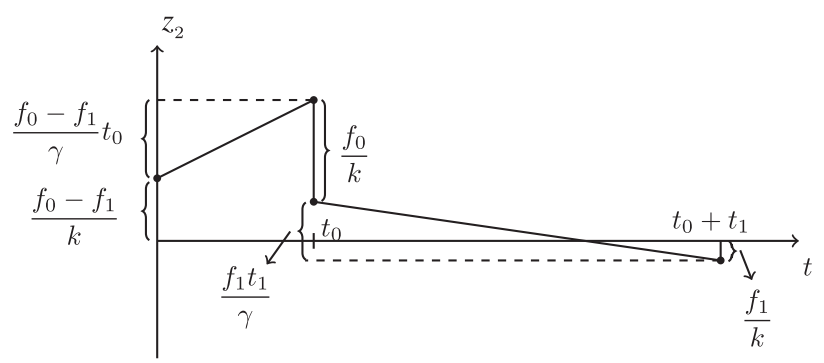

FIG. 4. The heuristic viscoelastic model used to discuss the enzyme cycle. (a) The Maxwell model of viscoelasticity: a spring of stiffness $k$ and dashpot of dissipation coefficient $\gamma$. $z_{2}$ is the position of the cylinder of the dashpot, $z_{1}$ the position of the piston of the dashpot. The force $f$ is applied to the cylinder. (b) The force $f$ vs time $t$ used to drive the cycle in the model [Eq. (3)]. $f_{0}$ represents the driving force due to the interaction between the substrate and the enzyme; it is turned on at $t=0$ when the substrate binds and turned off at $t=t_{0}$ when the reaction and/or product release occurs. $f_{1}$ is the internal restoring force of the enzyme (proportional to the free-energy gradient in the absence of substrate), always present for $z_{2} \neq 0$. For simplicity, $f_{0}$ and $f_{1}$ are taken as constants. The time interval $t_{1}$ is chosen such that the system returns to the initial state at $t=t_{0}+t_{1}$. (c) The corresponding response of the Maxwell element: $z_{2}(t)$ [Eq. (5)]. 
the substance. Now it is a simple matter to integrate Eq. (2) using Eq. (3) since

$$
\dot{f}=\left(f_{0}-f_{1}\right) \delta(t)-f_{0} \delta\left(t-t_{0}\right)+f_{1} \delta\left(t-t_{0}-t_{1}\right),
$$

and the result is [Fig. 4(c)]

$z_{2}= \begin{cases}\frac{f_{0}-f_{1}}{f_{1}}+\frac{f_{0}-f_{1}}{\gamma} t & \text { for } 0 \leq t<t_{0}, \\ -\frac{f_{1}}{k}+\frac{f_{0}-f_{1}}{\gamma} t_{0}-\frac{f_{1}}{\gamma} t & \text { for } t_{0} \leq t<t_{0}+t_{1}, \\ 0 & \text { otherwise, }\end{cases}$

where $t_{1}=\left(f_{0}-f_{1}\right) t_{0} / f_{1}$ ensures that the system is back in the original state at $t=t_{0}+t_{1}$. Evidently, we must have $f_{1}<f_{0}$. The "conformational change" is

$$
z_{\max }=z_{2}\left(t_{0}^{-}\right)=\frac{f_{0}-f_{1}}{k}\left(1+\frac{k}{\gamma} t_{0}\right) .
$$

In order to have the enzyme perform mechanical work, we may want to apply a load $F_{\mathrm{L}}$ for $t_{0} \leq t<t_{0}+t_{1}$; then $t_{1}$ is modified:

$$
t_{1}=\frac{f_{0}-f_{1}}{f_{1}-F_{\mathrm{L}}} t_{0}
$$

which diverges for $F_{\mathrm{L}} \rightarrow f_{1}$. In this scenario, it is the restoring force $f_{1}$ that performs the work, during the return (slow) part of the cycle, and evidently $f_{1}$ is the "stall force" for the enzyme. (See, for example, the working of the motor protein kinesin; a succinct summary is given in [27], which also contains references to the original literature.) The work supplied by the external force (arising from the binding of the substrate) is

$$
\begin{aligned}
W_{1} & =f_{0} z_{2}\left(t_{0}^{-}\right)=f_{0}\left(\frac{f_{0}-F_{\mathrm{L}}}{k}+\frac{f_{0}-F_{\mathrm{L}}}{\gamma} t_{0}\right) \\
& =\frac{f_{0}^{2}}{k}\left(1+\frac{k}{\gamma} t_{0}\right)\left(1-\frac{F_{\mathrm{L}}}{f_{0}}\right) .
\end{aligned}
$$

The work delivered to the dashpot (i.e., the energy dissipated) is

$$
\begin{aligned}
W_{2} & =\frac{\left(f_{0}-f_{1}\right)^{2}}{\gamma} t_{0}+\frac{\left(f_{1}-F_{\mathrm{L}}\right)^{2}}{\gamma} t_{1} \\
& =\left(f_{0}-f_{1}\right)\left(f_{1}-F_{\mathrm{L}}\right) \frac{t_{0}}{\gamma} \\
& \Rightarrow \frac{W_{2}}{W_{1}}=\frac{1-F_{\mathrm{L}} / f_{0}}{1+\gamma /\left(k t_{0}\right)},
\end{aligned}
$$

and the work done against the load is

$$
W_{3}=F_{\mathrm{L}} z_{2}\left(t_{0}^{-}\right)=F_{\mathrm{L}} z_{\max },
$$

$$
\Rightarrow \frac{W_{3}}{W_{1}}=\frac{F_{\mathrm{L}}}{f_{0}} .
$$

Alternatively, if we apply the load at $t_{0}^{+}$,

$W_{3}=F_{\mathrm{L}} z_{2}\left(t_{0}^{+}\right)=F_{\mathrm{L}}\left(z_{\max }-\frac{f_{0}}{k}\right) \Rightarrow \frac{W_{3}}{W_{1}}=\frac{F_{\mathrm{L}}}{f_{0}}-\frac{F_{\mathrm{L}}}{k z_{\max }}$.

Let us now look at some consequences of this model cycle:

Consequence 1. For $t_{1} \gg t_{0}$, the duration of the cycle is $\tau=t_{0}+t_{1} \approx t_{1}$, so the rate is [see Eq. (7)]

$$
\frac{1}{\tau} \approx \frac{f_{1}-F_{\mathrm{L}}}{f_{0}-f_{1}} \frac{1}{t_{0}},
$$

which goes to zero linearly with the load $F_{\mathrm{L}} ; f_{1}$ is evidently the stall force. This behavior is seen, for example, in the force-velocity curves of the motor protein kinesin [28].

Consequence 2. In the regime $t_{1}>t_{0}$, the maximum velocity of the enzyme is $\left(F_{L}=0\right)$

$$
v_{\max } \approx \frac{f_{1}}{\gamma}=\frac{F_{\text {stall }}}{\gamma}, \quad \text { i.e., } \quad \gamma \approx \frac{F_{\text {stall }}}{v_{\max }} .
$$

This result is not obvious: It relates the internal dissipation parameter of the protein to the stall force and the maximum velocity. (Notice that this relation is independent of "lever-arm length".) It could, in principle, be tested on any enzyme, and, if true, we would expect $F_{\text {stall }} / v_{\max }$ to be an approximately universal quantity based on our expectation that the dissipation parameter $\gamma$ is presumably not very different for different proteins. In other words, we expect fast enzymes (with large $v_{\max }$ ) to have "large" stall forces $F_{\text {stall }}$. Applied to kinesin, for which $F_{\text {stall }} \approx 5 \mathrm{pN}$ and $v_{\max } \approx 10 \mu \mathrm{m} / \mathrm{s}$ [29], we obtain $\gamma \approx 5 \times 10^{-4} \mathrm{~g} / \mathrm{s}$. Our measured value for GK [11] is $\gamma=4 \times 10^{-2} \mathrm{~g} / \mathrm{s}$ at $10 \mathrm{~Hz}$. However, at $1 \mathrm{kHz}$ (kinesin's rate), this value would be smaller because of "shear thinning" (a nonlinear effect). For example, the value of $\gamma$ measured in [30] for a different protein (lysozyme) and higher shear rate was 5 times smaller $\left(8 \times 10^{-3} \mathrm{~g} / \mathrm{s}\right)$. Evidently, other effects may limit $v_{\max }$ for kinesin (such as binding-unbinding rates to the microtubule), but our point is that perhaps even a complicated molecule like kinesin operates not too far from the regime described by Eq. (15).

Consequence 3. For enzymes that deliver work (such as motors), the efficiency in our language is $\eta=W_{3} / W_{1} \leq$ $F_{\mathrm{L}} / f_{0}$ [Eq. (12)]. Since $F_{\mathrm{L}}<f_{1}<f_{0}$, the efficiency is maximum for $f_{1}$ and $f_{0}$ as close as possible (and $\eta$ can, in principle, be approximately 1 ). On the other hand, $f_{1}=$ $f_{0} / 2$ is a special working point at which the cycle is symmetric under time reversal (fast-slow for $f_{1}<f_{0} / 2$, slow-fast for $\left.f_{1}>f_{0} / 2\right)$. See the diagram of Fig. 5(a), where the work done by the external force (substrate 

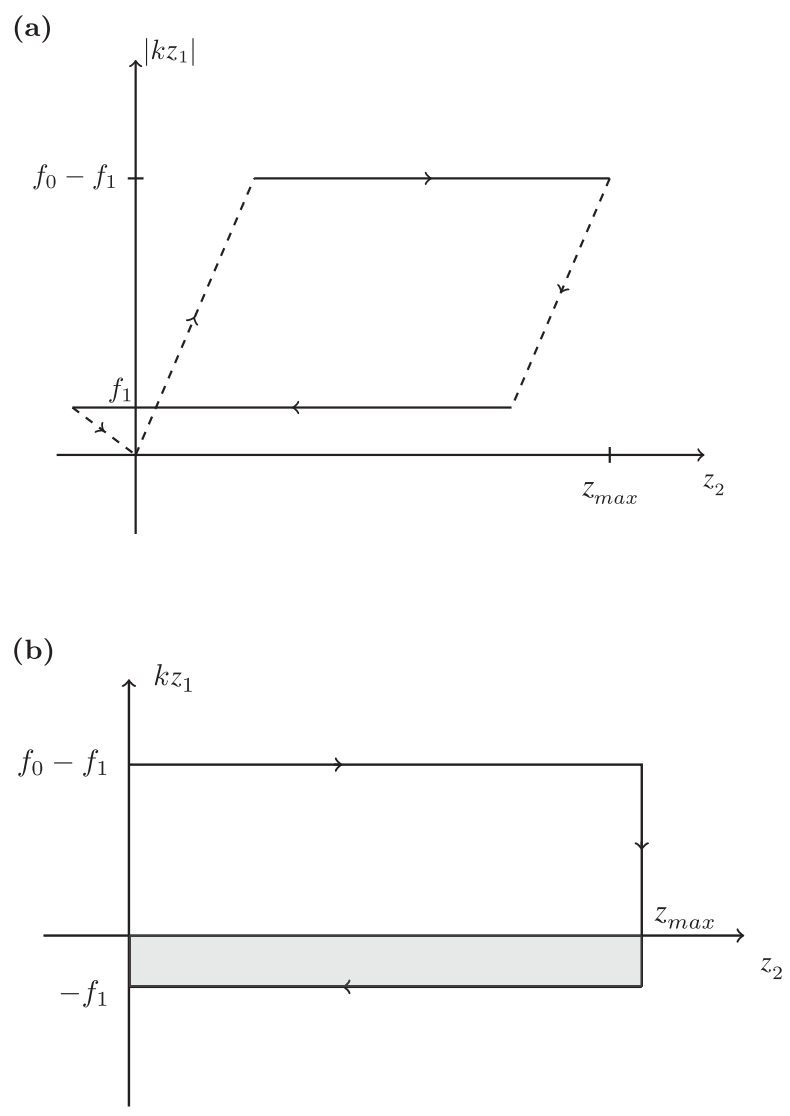

FIG. 5. (a) The "internal stress" $\left|k z_{1}\right|$ vs deformation $z_{2}$ for the model illustrated in Fig. 4. The dashed lines correspond to the jumps in the model. This cycle is qualitatively similar to the schematic cycle proposed in Fig. 3(a), which is itself suggested by the measurements of Fig. 2. (b) Simplified representation of the cycle in (a).

binding) is approximately $f_{0} z_{\max }$ and the maximum work one can extract is about $f_{1} z_{\max }$ (since $F_{L} \leq f_{1}$ ). Thus, the area of the cycle in the $\left(k z_{1}, z_{2}\right)$ plane [Fig. 5(b)] is the work done by the external force, while the shaded area in the figure is the work one can extract. So, while the efficiency can be up to $1\left(f_{1}=f_{0}\right)$, for a fast-slow cycle,

$$
f_{1} \leq \frac{f_{0}}{2} \Rightarrow \eta \leq 50 \% \text {. }
$$

Intriguingly, the actual efficiency of kinesin [31] is 50\%, so it is quite possible that kinesin works close to this regime. Indeed, let us suppose that

$$
f_{0} z_{\max } \approx \Delta G_{\text {bind }} \approx \Delta G_{\mathrm{r}}
$$

where $\Delta G_{\text {bind }}$ is the free energy of substrate binding and $\Delta G_{\mathrm{r}}$ the free energy of the reaction. [The first equality states that ATP binding is the actual driving force for the motor; the second equality states that the free energy of ATP hydrolysis removes the products from the binding site; both statements are probably roughly correct for kinesin.) Then, using $z_{\max }=8 \mathrm{~nm}$ (which is the step size of kinesin, i.e., we are reporting forces to the "lever arm") and $\Delta G_{\mathrm{r}}=13 \mathrm{kcal} / \mathrm{mol}=20 \mathrm{kT} /$ molecule, we find

$$
\begin{gathered}
f_{0} \approx \frac{\Delta G_{\mathrm{r}}}{z_{\max }}=\frac{20 \mathrm{kT}}{8 \mathrm{~nm}}=10 \mathrm{pN}, \\
f=F_{\text {stall }}=f_{0} / 2=5 \mathrm{pN},
\end{gathered}
$$

which is indeed the stall force of kinesin.

Consequence 4. What is special about the regime $f_{1}=f_{0} / 2$ ? The duration of the cycle is

$$
\tau=t_{0}+t_{1} \propto \frac{f_{0}}{f_{1}\left(f_{0}-f_{1}\right)}
$$

as far as force dependence is concerned. [We used Eqs. (14) and (16), with $F_{\mathrm{L}}=0, k t_{0} / \gamma \gg 1$, and $z_{\max }$ given.] Given $f_{0}$, from Eq. (19) $\tau$ is minimum for $f_{1}=$ $f_{0} / 2$; i.e., this condition maximizes the speed of the cycle.

Consequence 5. We may calculate the maximum rate for enzymes that operate in a cycle across the viscoelastic transition. In order to still have a cycle, $z_{\max }$ must be beyond the elastic limit at, say, $z_{\max } \approx 2 \AA$. The question is then: What is the maximum possible value for $f_{0}$ ? In a real physical system, the answer depends on the rate, as Fig. 2 shows. We may phrase the question as follows: What is the maximum stress the enzyme can sustain over a given time scale $\tau$ (or frequency $\nu=1 / \tau$ )? For an order-ofmagnitude estimate, we may use the result of Evans and Ritchie for the strength of molecular bonds [14]:

$$
F=\frac{T}{\delta} \ln \left(\frac{\nu}{\nu_{0}}\right)
$$

where $F$ is the bond-rupture force, $\nu$ the frequency, $\nu_{0}$ the zero-force unbinding rate, and $\delta$ a microscopic length scale (barrier position) which we identify approximately with our "critical deformation" $z_{\mathrm{c}}$ (which is also approximately the same as $z_{\max }$ in this regime). Thus we have, for the maximum stress sustainable at frequency $\nu$,

$$
f_{0}^{\max }\left(\nu_{2}\right)-f_{0}^{\max }\left(\nu_{1}\right)=\frac{T}{\delta} \ln \left(\frac{\nu_{2}}{\nu_{1}}\right) .
$$

Very roughly, from our own measurements on GK [11], the yield force at $10 \mathrm{~Hz}$ is $f_{0}^{\max }(10 \mathrm{~Hz}) \approx 10 \mathrm{pN}$, while $T / \delta=20 \mathrm{pN}$ for $\delta=2 \AA$. Thus, for example, at $\nu_{2}=$ $100 \mathrm{kHz}$, the maximum force is given by

$$
f_{0}^{\max }(100 \mathrm{kHz})=10 \mathrm{pN}+20 \times 4 \ln (10) \mathrm{pN} \approx 200 \mathrm{pN},
$$

and increases by about $50 \mathrm{pN}$ for each decade in frequency. Evidently, the numbers are such that this estimate gives $f_{0}^{\max } \approx 200 \mathrm{pN}$ in the relevant range of frequencies. (This value can also be extracted directly from Fig. 2 in [14].)

In fact, the relation (20) applies only for low forces [32-35]: 


$$
F \ll F^{*}=\Delta G /(\alpha \delta)
$$

[35], where $\Delta G$ is the barrier height, $\delta$ the barrier position, and $\alpha=2 / 3$, such that the shape of the barrier is not much perturbed. The more general theory of Dudko et al. [35] shows that, in fact, the rupture force increases faster than linearly with the log of the frequency. However, this effect does not change the order of magnitude of the estimate above, as can be seen from Fig. 2 in [35]. We may see the above estimate $f_{0}^{\max } \approx 200 \mathrm{pN}$ as a lower bound, while a higher bound is provided by the critical force $F^{*}$, at which the barrier vanishes [35]. Using reasonable values $\Delta G \approx$ $20 \mathrm{kT}$ (as in [35]), $\alpha=2 / 3$, and $\delta \approx 0.2 \mathrm{~nm}$ in the formula above gives $F^{*} \approx 600 \mathrm{pN}$. Then taking $f_{0}^{\max }=$ $400 \mathrm{pN}$, which is the median value between our rough lower bound $(200 \mathrm{pN})$ and upper bound $(600 \mathrm{pN})$ for this force, we obtain

$$
\left(\frac{1}{\tau}\right)_{\max } \approx \frac{f_{0}^{\max }}{4 \gamma z_{\max }} \approx \frac{400 \mathrm{pN}}{0.2 \mathrm{~nm} \times 4 \times 10^{-2} \mathrm{~g} / \mathrm{s}} \approx 50 \mathrm{kHz},
$$

using our value $\gamma=4 \times 10^{-2} \mathrm{~g} / \mathrm{s}$ [and using Eq. (19) with $f_{1}=f_{0} / 2$ ]. Once again, because of shear thinning, the relevant value of $\gamma$ is probably about a factor 10 smaller. (See also the measurement of $\gamma$ in [30].) Then the estimate (23) for the maximum rate of enzymes that perform conformational changes is close to the truth. (The fastest enzymes, such as carbonic anhydrase, have rates of about $1 \mathrm{MHz}$ but very small conformational motion [36]). This result is remarkable because the estimate (23) depends only on purely mechanical quantities.

\section{CONCLUSIONS}

In conclusion, we propose a nonequilibrium thermodynamic cycle based on the viscoelastic transition of the folded state of proteins [13] as one universal feature of enzyme action. "Thermodynamic" here means ensemble averaged. The cycle operates between two different speeds for the forward and backward paths. This possibility arises because the stress-strain curves of Fig. 2 are frequency dependent, which is, in our view, the same phenomenon discovered by Evans and collaborators in the context of bond-rupture forces [14]. The viscoelastic transition itself may correspond, in structural terms, to the previously proposed "cracking" [37,38]. In general, the approach we introduce here describes the dynamics through ensemble-averaged variables and is thermodynamic in this sense. In the present framework, several general properties of enzymes are easily understood or predicted. Among them, we find that the speed of molecular motors decreases linearly with load force [Eq. (15)]. We find a relation between the maximum speed $v_{\max }=\left(z_{\max } / \tau\right)_{\max }$ of an enzyme, its stall force $F_{\text {stall }}$, and the dissipation coefficient $\gamma$ [Eq. (15)]. Here, $z_{\max }$ is the amplitude of the conformational change on substrate binding and $1 / \tau$ the maximum rate (the rate under conditions where substrate binding is not rate limiting). Stall forces have hitherto been measured only for processive enzymes such as motors and polymerases, which are particularly complicated enzymes. We envision similar measurements in the future on simpler enzymes, such as GK; the experimental methods are available in principle. For example, mechanical stresses can be applied to an enzyme such as GK through molecular springs while, at the same time, monitoring the enzymatic activity, as described in Ref. [9]; or else oscillatory stresses can be applied to the enzyme in a nanorheology setup as described in Ref. [12], where it is also feasible (although not reported yet) to simultaneously monitor enzymatic activity. We can estimate the maximum rate of those enzymes that couple conformational motion to substrate binding or catalysis (which probably cover most enzymes) from purely mechanical parameters [Eqs. (21)-(23)]. The crucial quantity is the dissipation coefficient $\gamma$. Very few measurements have been taken of the "internal viscosity" of proteins, and the results differ by 8 orders of magnitude [11,39]. We argued in [11] that the reason for this variance is the viscoelastic nature of the protein's mechanics, which makes the internal viscosity frequency dependent $\left(\eta \sim 1 / \nu^{2}\right.$ at high frequency). The dissipation coefficient $\gamma$ is, on the other hand, not frequency dependent (except for nonlinear effects such as shear thinning, but this dependence probably is not dramatic), and we show in the present work that the value $\gamma \sim 10^{-2} \mathrm{~g} / \mathrm{s}$, obtained experimentally first by Radmacher et al. [30] and later by us [11], leads to a consistent picture of the dynamics of proteins. Evidently, the precise value of $\gamma$, which is an effective parameter, will depend on the specific protein and conformational change; here, we are concerned with the order of magnitude. The other crucial quantity that enters the estimate of the maximum rate of enzymes is the maximum stress that the enzyme can sustain over a given time. Here we are in a better position to give an estimate, because the related problem of bondrupture forces has been studied extensively [14,32,34]; it appears that $f_{0}^{\max } \approx 400 \mathrm{pN}$ is, within a factor 2 , a reasonable value at the relevant frequencies.

A different aspect of this work is that the nonequilibrium cycle we propose provides a basic way of breaking timereversal symmetry (for $f_{1} \neq f_{0} / 2$ in the simple model that we consider) in the cycle of the enzyme itself, which is, after all, necessary to obtain a molecular machine. This aspect is connected with the question of obtaining directed motion from a molecular machine, which has also been studied extensively [40]. On the other hand, we find that the regime $f_{1}=f_{0} / 2$, which corresponds to the cycle proceeding at the same speed in the forward and backward directions, has several special features (such as maximizing the rate), so $f_{1} / f_{0}$ (in this language) may be a parameter that, for different enzymes, is tuned to different values between 0 and 1 . 
Thermal fluctuations of the thermodynamic variables are conspicuously absent from our discussion. One might argue that, if $f_{0} z_{\max } \gg \mathrm{kT}$, this absence is not too serious a shortcoming in our model, but in fact it is one question that will have to be addressed in future work.

Finally, we remark that, taken individually, several of the ideas presented here have been put forward before. For instance, a linear relationship between force and velocity ( $v=F / \gamma$, which applies in the viscoelastic regime) was invoked in [41] to obtain the experimentally observed linear behavior of motor-protein velocity with load. Similarly, the fact that conformational motion of enzymes must operate beyond the linear elasticity regime has been understood before [38,42]. And, more generally, the present model is essentially an explicit representation of Blumenfeld's relaxation model [4], based on experimental facts (the viscoelastic transition) that were not known at the time.

So what we contribute that is new is the direct experimental evidence of the viscoelastic nature of the protein's mechanics (Fig. 2) and the consequent realization that, because of the frequency dependence of this viscoelastic transition, a nonequilibrium cycle can open between the forward and backward conformational changes if they happen at different speeds. Our work provides a framework for considering mechanochemical coupling in enzymes based on universal materials properties rather than the specific structure of the folded state.

\section{ACKNOWLEDGMENTS}

This work was supported by the UC Lab Research Program and by NSF Grant No. DMR-1006162. We thank Jonathan Landy for comments on the manuscript.

[1] D. E. Koshland, Jr., Application of a Theory of Enzyme Specificity to Protein Synthesis, Proc. Natl. Acad. Sci. U.S.A. 44, 98 (1958).

[2] J. Monod, J.-P. Changeux, and F. Jacob, Allosteric Proteins and Cellular Control Systems, J. Mol. Biol. 6, 306 (1963).

[3] W.S. Bennett, Jr. and T. A. Steitz, Glucose-Induced Conformational Change in Yeast Hexokinase, Proc. Natl. Acad. Sci. U.S.A. 75, 4848 (1978).

[4] L.A. Blumenfeld and A.N. Tikhonov, Biophysical Thermodynamics of Intracellular Processes: Molecular Machines of the Living Cell (Springer Verlag, New York, 1994).

[5] M. J. Schnitzer and S. M. Block, Kinesin Hydrolyses One ATP per 8-nm Step, Nature (London) 388, 386 (1997).

[6] H. Noji, R. Yasuda, M. Yoshida, and K. Kinosita, Jr., Direct Observation of the Rotation of $\mathrm{F}_{1}$-ATPase, Nature (London) 386, 299 (1997).

[7] B. Choi, G. Zocchi, Y. Wu, S. Chan, and L. J. Perry, Allosteric Control through Mechanical Tension, Phys. Rev. Lett. 95, 078102 (2005).
[8] Y. Savir and T. Tlusty, Conformational Proofreading: The Impact of Conformational Changes on the Specificity of Molecular Recognition, PLoS ONE 2, e468 (2007).

[9] C-Y. Tseng, A. Wang, and G. Zocchi, Mechano-chemistry of the Enzyme Guanylate Kinase, Europhys. Lett. 91, 18005 (2010).

[10] A. Fersht, Structure and Mechanism in Protein Science: A Guide to Enzyme Catalysis and Protein Folding (Freeman, San Francisco, 1998).

[11] Y. Wang and G. Zocchi, The Folded Protein as a Viscoelastic Solid, Europhys. Lett. 96, 18003 (2011).

[12] Y. Wang and G. Zocchi, Elasticity of Globular Proteins Measured from the ac Susceptibility, Phys. Rev. Lett. 105, 238104 (2010). In that paper, we introduced the nanorheology method with which the data of Fig. 2 of the present paper were obtained. GNPs are tethered to a flat surface by the enzyme under study. The collective oscillation of the GNPs, driven by an oscillating electric field, is detected by evanescent wave scattering using homodyne (lock-in) detection. Approximately $10^{7}$ GNPs contribute to the scattered light; thus, the signal represents the ensemble-averaged amplitude of oscillation of the GNPs. We demonstrate the method by detecting the change in stiffness of the enzyme GK upon binding the substrate GMP.

[13] Y. Wang and G. Zocchi, Viscoelastic Transition and Yield Strain of the Folded Protein, PLoS ONE 6, e28097 (2011).

[14] E. Evans and K. Ritchie, Strength of a Weak Bond Connecting Flexible Polymer Chains, Biophys. J. 76, 2439 (1999).

[15] S. Sacquin-Mora, O. Delalande, and M. Baaden, Enzyme Closure and Nucleotide Binding Structurally Lock Guanylate Kinase, Biophys. J. 101, 1440 (2011).

[16] Y. Li, Y. Zhang, and H. Yan, Kinetic and Thermodynamic Characterizations of Yeast Guanylate Kinase, J. Biol. Chem. 271, 28038 (1996).

[17] H. A. Kramers, Brownian Motion in a Field of Force and the Diffusion Model of Chemical Reactions, Physica (Amsterdam) 7, 284 (1940).

[18] G. I. Bell, Models for the Specific Adhesion of Cells to Cells, Science 200, 618 (1978).

[19] F. Jülicher, A. Ajdari, and J. Prost, Modeling Molecular Motors, Rev. Mod. Phys. 69, 1269 (1997).

[20] K. Henzler-Wildman and D. Kern, Dynamic Personalities of Proteins, Nature (London) 450, 964 (2007).

[21] S. J. Benkovic, G. G. Hammes, and S. Hammes-Schiffer, Free-Energy Landscape of Enzyme Catalysis, Biochemistry 47, 3317 (2008).

[22] L. A. Blumenfeld, Activation Parameters of Enzyme Reactions (Applicability of the Activated Complex Theory in Enzymology) (in Russian), Biofizika 16, 724 (1971), http://www.ncbi.nlm.nih.gov/pubmed/5571253.

[23] L. A. Blumenfeld, Elementary Steps in Enzyme Catalysis (in Russian), Biofizika 17, 954 (1972), http:// www.ncbi.nlm.nih.gov/pubmed/4643725.

[24] E. Z. Eisenmesser, D. A. Bosco, M. Akke, and D. Kern, Enzyme Dynamics During Catalysis, Science 295, 1520 (2002).

[25] H. Qu, J. Landy, and G. Zocchi, Cracking Phase Diagram for the Dynamics of an Enzyme, Phys. Rev. E 86, 041915 (2012). 
[26] L.A. Blumenfeld, The Physical Aspects of Enzyme Functioning, J. Theor. Biol. 58, 269 (1976).

[27] K. Sneppen and G. Zocchi, Physics in Molecular Biology (Cambridge University Press, Cambridge, England, 2005).

[28] K. Svoboda and S. M. Block, Force and Velocity Measured for Single Kinesin Molecules, Cell 77, 773 (1994).

[29] K. Visscher, M. J. Schnitzer, and S. M. Block, Force Production by Single Kinesin Motors, Nat. Cell Biol. 2, 718 (2000).

[30] M. Radmacher, M. Fritz, J. P. Cleveland, D. A. Walters, and P. K. Hansma, Imaging Adhesion Forces and Elasticity of Lysozyme Adsorbed on Mica with the Atomic Force Microscope, Langmuir 10, 3809 (1994).

[31] J. Howard, The Movement of Kinesin Along Microtubules, Annu. Rev. Physiol. 58, 703 (1996).

[32] U. Seifert, Dynamic Strength of Adhesion Molecules: Role of Rebinding and Self-Consistent Rates, Europhys. Lett. 58, 792 (2002).

[33] G. Hummer and A. Szabo, Kinetics from Nonequilibrium Single-Molecule Pulling Experiments, Biophys. J. 85, 5 (2003).

[34] O. K. Dudko, A. E. Filippov, J. Klafter, and M. Urbakh, Beyond the Conventional Description of Dynamic Force Spectroscopy of Adhesion Bonds, Proc. Natl. Acad. Sci. U.S.A. 100, 11378 (2003).

[35] O. K. Dudko, G. Hummer, and A. Szabo, Intrinsic Rates and Activation Free Energies from Single-Molecule
Pulling Experiments, Phys. Rev. Lett. 96, 108101 (2006).

[36] P. Hammarström, R. Owenius, L. G. Mårtensson, U. Carlsson, and M. Lindgren, High-Resolution Probing of Local Conformational Changes in Proteins by the Use of Multiple Labeling: Unfolding and Self-Assembly of Human Carbonic Anhydrase II Monitored by Spin, Fluorescent, and Chemical Reactivity Probes, Biophys. J. 80, 2867 (2001).

[37] O. Miyashita, J. N. Onuchic, and P. G. Wolynes, Nonlinear Elasticity, Proteinquakes, and the Energy Landscapes of Functional Transitions in Proteins, Proc. Natl. Acad. Sci. U.S.A. 100, 12570 (2003).

[38] P.C. Whitford, O. Miyashita, Y. Levy, and J. N. Onuchic, Conformational Transitions of Adenylate Kinase: Switching by Cracking, J. Mol. Biol. 366, 1661 (2007).

[39] K. W. Plaxco and D. Baker, Limited Internal Friction in the Rate-Limiting Step of a Two-State Protein Folding Reaction, Proc. Natl. Acad. Sci. U.S.A. 95, 13591 (1998).

[40] M. O. Magnasco, Forced Thermal Ratchets, Phys. Rev. Lett. 71, 1477 (1993).

[41] C. Bustamante, D. Keller, and G. Oster, The Physics of Molecular Motors, Acc. Chem. Res. 34, 412 (2001).

[42] J. A. McCammon, B. R. Gelin, and M. Karplus, Dynamics of Folded Proteins, Nature (London) 267, 585 (1977). 